\title{
Brown Bowel Syndrome Is a Rare and Commonly Missed Disease: A Case Report and Literature Review
}

\author{
Joaquin Ponce-Zepeda ${ }^{D}$, , Wenchang Guo ${ }^{1}{ }^{1},{ }^{1}$ Giorgioni Carmen ${ }^{1},{ }^{1}$ Daniel Moon Kim $(\mathbb{D})^{2}$ \\ Gregory C. Albers $\mathbb{D}^{2},{ }^{2}$ Vishal Suresh Chandan $\mathbb{D D}^{1},{ }^{1}$ and Xiaodong Li $\mathbb{i D}^{1}$
}

${ }^{1}$ Department of Pathology and Laboratory Medicine, University of California-Irvine, Irvine, CA, USA

${ }^{2}$ Department of Internal Medicine, Gastroenterology, University of California-Irvine, Irvine, CA, USA

Correspondence should be addressed to Xiaodong Li; xiaodol4@uci.edu

Received 13 October 2020; Revised 27 January 2021; Accepted 27 February 2021; Published 5 March 2021

Academic Editor: Daniel C. Damin

Copyright (C) 2021 Joaquin Ponce-Zepeda et al. This is an open access article distributed under the Creative Commons Attribution License, which permits unrestricted use, distribution, and reproduction in any medium, provided the original work is properly cited.

\begin{abstract}
Background. Brown bowel syndrome (BBS) is a rare gastrointestinal condition, and vitamin E deficiency has been considered to be a main contributor. However, vitamin E deficiency has been found in only a few patients throughout the published literature studies and its cutoff lab value for diagnosis is not entirely clarified. Case Presentation. A 56-year-old female patient with a history of congenital bowel obstruction (repaired at birth) presented with bloating, abdominal pain, and chronic diarrhea. Endoscopy identified unremarkable gastrointestinal mucosa except a few small polyps in the colon. A partial obstruction was detected by a small bowel follow-through series and then confirmed by CT scan. The resected small bowel was significantly dilated with a thickened brown wall and extensive serosal adhesion. Microscopic examination revealed unremarkable mucosa, but dense granular brown pigments were identified in the cytoplasm of the smooth muscle cells in the muscularis propria. These deposits resulted to be lipofuscin, and BBS was diagnosed. The patient was asymptomatic at 9-month follow-up after surgery without vitamin E supplement. Conclusion. Mitochondrial damage with lipofuscin deposition is at the root of BBS pathogenesis. Any etiology associated with mitochondrial damage can cause this disease, and vitamin E deficiency is just one of them. Dysmotility from extensive serosal adhesion could be a possible etiology for this patient. Due to overlapping symptoms, lipofuscin deposition primarily in the muscularis propria, and unclear serum value of vitamin E, this syndrome is often missed in routine clinical practice from the superficial biopsy. A transmural biopsy is necessary for a definite diagnosis.
\end{abstract}

\section{Introduction}

Brown discoloration accumulating in the human intestinal wall secondary to lipofuscin was first observed by Wagner in 1861 [1], and the terminology of brown bowel syndrome (BBS) was formally introduced by Toffler in 1963 [2]. BBS is a rare gastrointestinal condition, and less than 70 cases have been reported in the literature. Since this syndrome usually occurred in patients with underlying conditions causing chronic long-term or severe malabsorption, vitamin $\mathrm{E}$ deficiency has been accordingly considered to be a main contributor. However, vitamin E deficiency has been confirmed in only a few patients throughout these literature studies, and its cutoff lab value for diagnosis is not still clarified.

\section{Case Presentation}

A 56-year-old female presented with bloating, abdominal pain, and chronic diarrhea for one year and weight loss of 20 pounds. As per the patient, she had a congenital bowel obstruction repaired at birth; however, the official details were not documented in a formal medical report. Her family history included diverticulitis, colitis, and colon cancer on her maternal side. Endoscopic examination showed unremarkable mucosa of both stomach and duodenum; colonoscopy identified two small polyps in the distal transverse colon and proximal descending colon. A small bowel followthrough was further conducted and revealed relatively highgrade partial mechanical bowel obstruction. The following 
CT scan confirmed the small bowel obstruction along with marked hepatic steatosis.

An open exploration revealed a segment of small bowel with marked dilation and extensive adhesions to the abdominal wall. A resected segment of the small bowel $(35.5 \mathrm{~cm}$ in length) showed an extensive fibrotic and adhesive serosal surface, which was significantly dilated at the proximal end $(11.0 \mathrm{~cm}$ in greatest diameter $)$. Upon sectioning, the small bowel mucosa was unremarkable, and the wall of the small bowel was distinctly thickened (measuring $0.6-0.8 \mathrm{~cm}$ ) and appeared dark brown (Figure 1(a)). Microscopic examinations revealed prominent hypertrophy of the muscularis propria with normal small bowel mucosa. However, the fine granular brown pigments were identified to be confined in the cytoplasm of the smooth muscle cells in the muscularis propria, not in muscular mucosae (Figures 1(b) and 1(c)). Special stains were performed, and these granular deposits were negative for iron and trichrome, strongly positive for periodic acid-Schiff (PASD) (Figure 1(d)), and patchy positive for Fontana-Masson in both smooth muscle cells in the muscularis propria (Figure 1(e)) and blood vessels (Figure 1(f)). These granular deposits resulted to be lipofuscin by these special stains. The immunohistochemistry (IHC) for CD117, calretinin, and S100 revealed normal interstitial cells of Cajal, nerve cells, and ganglion cells and ruled out neuropathic disease. An infection process has also been ruled out by both normal mucosa morphology and negative specific stains for Gomori methenamine silver (GMS), PAS, and acid-fast bacilli (AFB). Celiac disease and inflammatory bowel disease (IBD) have been excluded based on both normal endoscopic and histologic mucosa. Severe malnutrition is unlikely due to normal hemoglobin $(11.8 \mathrm{G} / \mathrm{DL})$ and albumin $(4.2 \mathrm{G} / \mathrm{DL})$. Ultimately, BBS was the final diagnosis, and the patient was discharged without vitamin E supplement. She was closely followed up and remained asymptomatic as of the date of this manuscript writing.

\section{Discussion}

3.1. Pathogenesis. BBS is histologically characterized by pathologic lipofuscin accumulation in the cytoplasm of smooth muscle cells in the gastrointestinal wall [3]. Lipofuscin is a lysosomal degradation product of damaged mitochondria due to oxidative stress (an imbalance between antioxidant defenses and reactive oxygen species) [4]. In BBS patients, lipofuscin is confined in smooth muscle cells primarily in the muscularis propria, but also can be seen in the muscular mucosae and blood vessel wall. The remaining cells of the intestine including epithelial cells are not affected [5]. This accumulation can be seen in any part of the gastrointestinal wall including the appendix and gallbladder [3]; however, it shows the propensity to the small intestine.

Vitamin E deficiency has been considered to be a main contributor for the pathogenesis of BBS based on the following three major facts: First, vitamin E has antioxidative property and its deficiency has been proved to cause damage to the phospholipid layer of the mitochondrial membrane
[6], which in turn leads to the pathologic accumulation of lipofuscin $[7,8]$. Secondly, BBS usually occurs in patients with malabsorptive disorders that may cause vitamin $\mathrm{E}$ deficiency: celiac disease $[9,10]$, IBD [11], cystic fibrosis [12], liver disease [12, 13], chronic pancreatitis [10], motility disorders [14-16], and postoperative conditions (obesity/ bypass surgery) $[6,12,17]$. Third, some BBS patients were identified to have deficient vitamin $\mathrm{E}$ levels and showed an improvement in clinical symptoms after vitamin E supplementation $[1,12,15,18]$. However, these findings have been reported in only a few cases $[1,12,15,18]$ (for example, only one out of six patients in a recently published study [12]) and there is no literature to define the cutoff value of vitamin $\mathrm{E}$ for diagnosis and therapy. Other antioxidants, such as glutathione peroxidase, have been also found depleted in a patient with BBS [15]. Therefore, a large prospective study to clarify the oxidative stress, not only limited to vitamin $\mathrm{E}$, is necessary for the understanding of this disease.

Mitochondrial damage with lipofuscin deposition is the core of the pathogenesis of BBS. In this literature review, the emphasis is placed on a positive and continuous feedback loop between oxidative stress, damaged mitochondria, and lipofuscin deposition. Lipofuscin deposition, as a product of damaged mitochondria, also inhibits the effective degradation of oxidatively modified proteins [4]. Lipofuscin in smooth muscle cells further disrupts the muscle fiber architecture by interrupting the contractile myofibrils and finally causes dysmotility and eventually muscularis propria hypertrophy [19]. The resulting dysmotility in the bowel increases oxidative stress and further causes mitochondrial dysfunction. The patient in this report did not show obvious malnutrition, and the underlying disorders causing malnutrition, such as IBD, celiac disease, and chronic pancreatitis, have been excluded. The liposuction is primarily deposited in the adhesive small bowel segment, not the whole intestine. It was intestinal dysmotility that initiated this positive feedback loop of pathogenesis (shown in Figure 2).

3.2. Clinical Symptoms. Besides symptoms from the underlying disorders, BBS patients, like this case report, usually present with intestinal motility disorders (e.g., intestinal dilation, volvulus, and intussusception) [14, 20, 21]. Intestinal bleeding, an uncommon but fatal symptom, is another symptom secondary to lipofuscin in vascular walls [14]. Some patients may also present with extraintestinal manifestations of the syndrome including myopathy and neuromuscular dysfunction [1]. Only a few cases of carcinoma have been reported in BBS patients [22-24]. These occasional reports of carcinoma are most likely a concurrent carcinoma arising from underlying disorders (such as celiac disease and IBD) because carcinoma arises from epithelial cells, but the lipofuscin coalesces in the smooth muscle cells (not the epithelial cells) [5].

3.3. Diagnosis. BBS has been often missed or ignored due to several reasons. First, the incidence of the disease is rare (the level of suspicion is accordingly low). Second, the clinical 


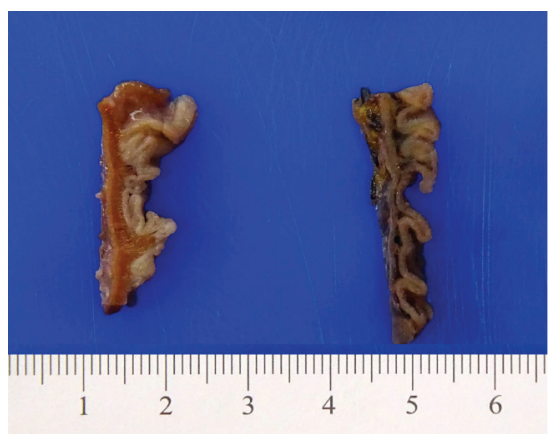

(a)

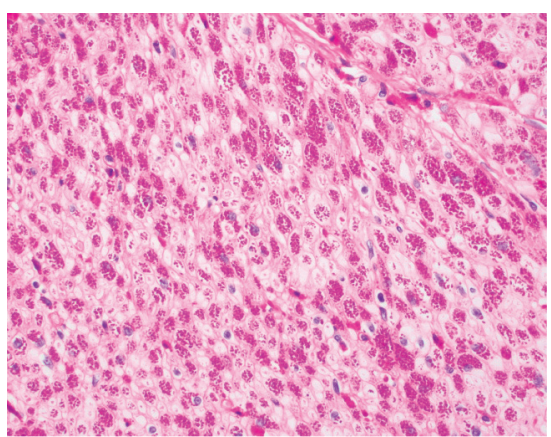

(d)

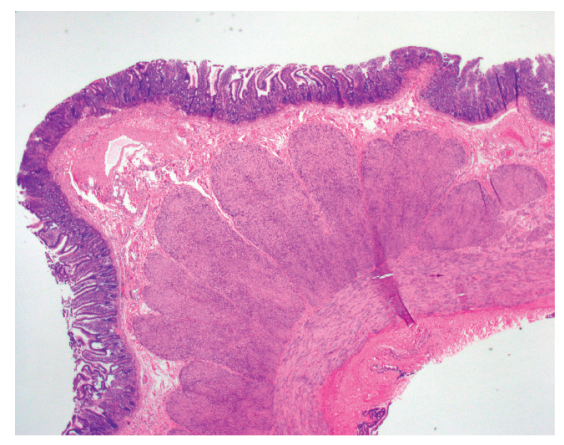

(b)

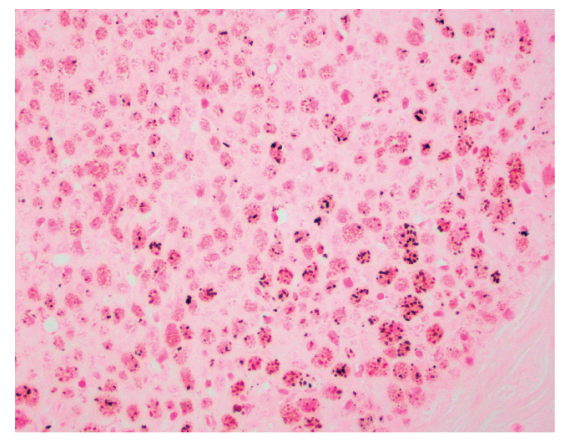

(e)

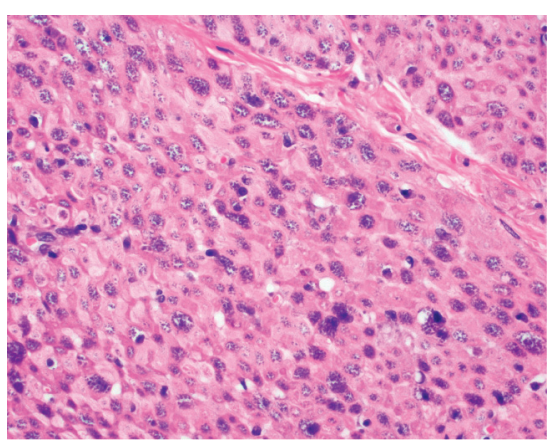

(c)

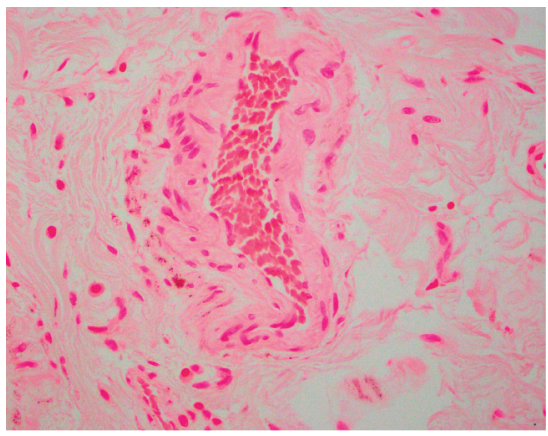

(f)

Figure 1: (a) Gross photos of small bowel from BBS (left) and from trauma patient as control (right). The wall is thicker and brown in comparison with the right. (b) H\&E stain (x2) showing brown deposits in the muscularis propria. (c) H\&E stain (x40) showing brown deposits in smooth muscle cells of the muscularis propria. (d) PASD stain (x40) highlighting brown deposits in the cytoplasm of smooth muscle cells. (e) Fontana-Masson stain (x40) highlighting brown deposits in the cytoplasm of smooth muscle cells. (f) Fontana-Masson stain (x40) highlighting brown deposits in smooth muscle cells of the blood vessel.

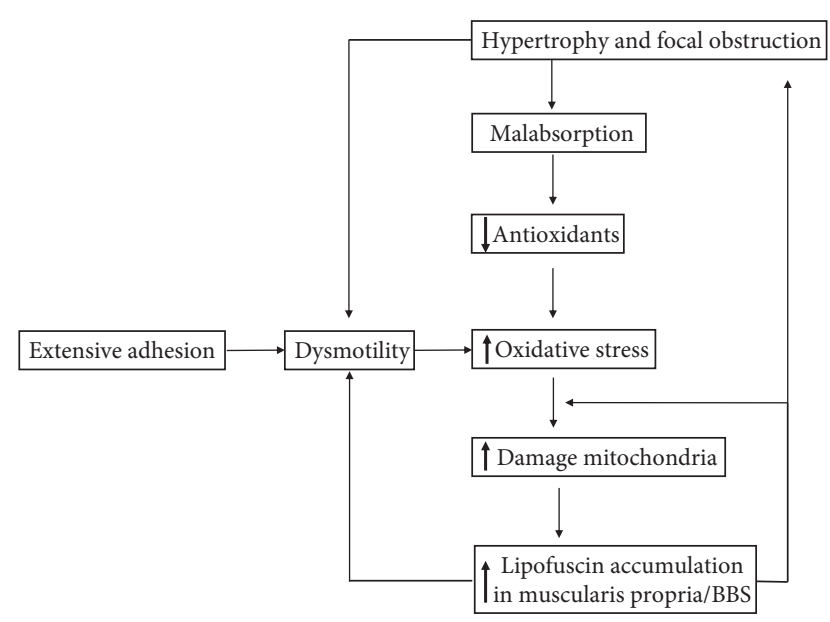

FIGURE 2: The positive feedback loop of the pathogenesis of BBS in this patient.

symptoms are not specific (BBS can be secondary to and coexist with the underlying diseases). Third, besides underlying disease, the endoscopic findings are unremarkable since lipofuscin accumulations are mainly in the muscularis propria which are not usually biopsied. Fourth, vitamin E cutoff value for diagnosis has not been established though its deficiency has been overestimated. Therefore, the transmural nature of the biopsy is critical for a definitive diagnosis. The key features for differential diagnosis are shown in Table 1.

3.4. Therapy and Prognosis. The treatment of BBS depends on the causes and the severity of symptoms. The patient with severe acute complications, such as bleeding or acute obstruction, usually requires immediate surgical intervention [1]. If BBS is found early or incidentally, the therapy is primarily conservative and includes treating the underlying malabsorption. Vitamin E supplementation usually takes months to show the benefits $[1,12,17]$, and caution must be taken because high doses can surpass its antioxidant properties and begin to work as a pro-oxidant $[25,26]$. The application of MitoTEMPO [4] or melatonin [27] has been also shown to prevent the accumulation of the lipofuscin protein aggregate and may be a potential novel therapy of targeting antioxidants to mitochondria. The prognosis of $\mathrm{BBS}$ is not clear due to rare cases reported; however, in a recent study [12], only one of eight patients died due to encephalitis with a mean clinical follow-up of 208 weeks (range: 10 to 770 ).

There is no definite proof of malnutrition, and dysmotility has been considered as the main cause in this BBS patient. The treatment for this patient included surgical procedure and close follow-up; however, vitamin E supplement was not provided. The patients did not show any 
TABLe 1: The key features of BBS for differential diagnosis.

Brown bowel syndrome

(1) Normal mucosa detected by endoscopy and histology (if no overlapping disease)

(2) PAS-positive pigments in smooth muscle cells of the muscularis propria

(3) EM: osmophilic pigments

Infection (Whipple disease and mycobacterium)

(1) Traveling or close contacting history

(2) Abnormal endoscopic findings

(3) PAS-positive granules in macrophages in lamina propria

(4) IHC for microorganism

(5) Normal muscularis propria

(6) EM: bacteria

Melanosis coli or duodeni

(1) Usually no clinical symptoms

(2) History of usage of anthraquinone laxatives or renal disease

(3) Dark endoscopic mucosa

(4) PAS-positive granules in macrophages in lamina propria

Celiac disease

(1) Inflamed duodenum detected by an endoscopy: scalloping or flattening of duodenal folds, fissuring over the folds, and a mosaic pattern of mucosa of folds

(2) Destruction of mucosa by intraepithelial lymphocytes

(3) Positive serology: antitransglutaminase antibodies, antiendomysial antibodies, and antigliadin antibodies

(4) Response to gluten-free products

Inflammatory bowel disease

(1) Bloody diarrhea

(2) Abnormal endoscopic findings: inflammation, ulcer, and loss of vascularity

(3) Active and chronic inflammation in mucosa or granuloma and transmural inflammation (Crohn's disease)

(4) No pigments seen

symptoms up to the date of this manuscript writing. The motility test (small bowel follow-through) and the detection of antioxidants such as thiamine and vitamins $\mathrm{A}$ and $\mathrm{E}$ have been suggested as additional studies in the evaluation of this patient.

\section{Conclusion}

BBS is a rare gastrointestinal disease usually associated with various chronic underlying diseases. Mitochondrial damage with lipofuscin deposition due to oxidative stress is the main process occurring in the pathogenesis of BBS. There are many factors at play in the pathogenesis of BBS, and any one of these factors, if involved, can cause BBS, whereas vitamin E deficiency is simply one of these factors. In this case, the dysmotility from extensive serosal adhesion could be a possible etiology.

Due to overlapping symptoms and deposits of lipofuscin within the muscularis propria, this disease has often been missed. BBS patients usually present with intestinal motility disorders, and a transmural biopsy is necessary to make a definitive diagnosis.

\section{Data Availability}

The figure data used to support the findings of this study are included within the article.

\section{Conflicts of Interest}

The authors declare that they have no conflicts of interest.

\section{Authors' Contributions}

All authors were involved in concept, design, writing, or revision of the manuscript.

\section{References}

[1] R. Parente, M. Pinamonti, and S. Martina, "Brown bowel syndrome in a middle-aged woman with chronic idiopathic malabsorption," Case Reports in Surgery, vol. 2019, Article ID 4706592, 2019.

[2] A. H. Toffler, P. B. Hukill, and H. M. Spiro, "Brown bowel syndrome," Annals of Internal Medicine, vol. 58, no. 5, pp. 872-877, 1963.

[3] G. A. Herrera, E. A. Turbat-Herrera, and V. G. Lockard, "Unusual pigmented vesical lesion in a middle-aged woman," Ultrastructural Pathology, vol. 14, no. 6, pp. 529-535, 1990.

[4] J. König, C. Ott, M. Hugo et al., "Mitochondrial contribution to lipofuscin formation," Redox Biology, vol. 11, pp. 673-681, 2017.

[5] E. Kaiserling, R. Schäffer, and J. Weckermann, "Brown bowel syndrome with manifestation in the gastrointestinal tract and thyroid gland," Pathology-Research and Practice, vol. 183, no. 1, pp. 65-74, 1988.

[6] H. Lee, A. M. Carlin, A. H. Ormsby, and M. W. Lee, "Brown bowel syndrome secondary to jejunoileal bypass: the first case report," Obesity Surgery, vol. 19, no. 8, pp. 1176-1179, 2009.

[7] Vitamin E, "Deficiency in dogs," Canadian Journal of Comparative Medicine and Veterinary Science, vol. 4, no. 4, p. 117, 1940.

[8] D. O. Cordes and A. H. Mosher, "Brown pigmentation (lipofuscinosis) of canine intestinal muscularis," The Journal 
of Pathology and Bacteriology, vol. 92, no. 1, pp. 197-206, 1966.

[9] C. E. Connolly, M. Kennedy, F. M. Stevens, and C. F. McCarthy, "Brown bowel syndrome occurring in coeliac disease in the west of Ireland," Scandinavian Journal of Gastroenterology, vol. 29, no. 1, pp. 91-94, 1994.

[10] H. Reynaert, S. Debeuckelaere, B. De Waele, M. Meysman, A. Goossens, and G. Devis, "The Brown bowel syndrome and gastrointestinal adenocarcinoma," Journal of Clinical Gastroenterology, vol. 16, no. 1, pp. 48-51, 1993.

[11] J. R. Lambert, S. C. Luk, and K. P. Pritzker, "Brown bowel syndrome in Crohn's disease," Archives of Pathology \& Laboratory Medicine, vol. 104, no. 4, pp. 201-205, 1980.

[12] C. A. Arnold, A. P. Burke, E. Calomeni et al., "Brown bowel syndrome," American Journal of Surgical Pathology, vol. 44, no. 6, pp. 834-837, 2020.

[13] C. N. Lin, A. H. Huang, S. I Hsu et al., "Brown bowel syndrome: report of two cases," Journal of the Formosan Medical Association = Taiwan Yi Zhi, vol. 92, no. 12, pp. 1090-1094, 1993.

[14] C. Ruchti, S. Eisele, and M. Kaufmann, "Fatal intestinal pseudo-obstruction in brown bowel syndrome," Archives of Pathology \& Laboratory Medicine, vol. 114, no. 1, pp. 76-80, 1990.

[15] H. C. Ward, J. Leake, P. J. Milla, and L. Spitz, "Brown bowel syndrome: a late complication of intestinal atresia," Journal of Pediatric Surgery, vol. 27, no. 12, pp. 1593-1595, 1992.

[16] G. Oberhuber, R. Pointner, E. Lauer, P. Waldenberger, and T. Radaszkiewicz, "Brown bowel” syndrome--lipofuscinosis of the intestine as a cause of atonia," Leber Magen Darm, vol. 19, no. 5, pp. 270-274, 1989.

[17] P. F. D. C. Soares, R. B. de Carvalho, E. A. Chaim, and E. Cazzo, "Brown bowel syndrome: a rare malnutrition-related complication of bariatric surgery," Nutricion Hospitalaria, vol. 36, no. 3, pp. 743-747, 2019.

[18] S. P. Lee and G. I. Nicholson, "Ceroid enteropathy and vitamin E deficiency," The New Zealand Medical Journal, vol. 83, no. 563, pp. 318-320, 1976.

[19] E. J. Feeney, S. Austin, and Y. H. Chien, "The value of muscle biopsies in Pompe disease: identifying lipofuscin inclusions in juvenile-and adult-onset patients," Acta Neuropathologica Communications, vol. 2, p. 2, 2014.

[20] A. Anand and B. M. Jaffe, "Brown bowel syndrome: case report and review," Journal of Louisiana State Medical Society, vol. 157 , no. 6 , pp. $338-340,2005$

[21] C. S. Foster, "The brown bowel syndrome: a possible smooth muscle mitochondrial myopathy?" Histopathology, vol. 3, no. 1, pp. 1-17, 1979.

[22] M. Raithel, T. T. Rau, and A. F. Hagel, "Jejunitis and brown bowel syndrome with multifocal carcinogenesis of the small bowel," World Journal of Gastroenterology, vol. 21, no. 36, pp. 10461-10467, 2015.

[23] M. Shiller, I. Cohen, M. Munichor, N. Loberant, A. Bickel, and R. Reshef, "The "brown bowel syndrome" associated with jejunal carcinoma," American Journal of Gastroenterology, vol. 88, no. 10, pp. 1788-1789, 1993.

[24] H. Reynaert and G. Devis, "The brown bowel syndrome and gastrointestinal adenocarcinoma," The American Journal of Gastroenterology, vol. 89, no. 5, pp. 812-813, 1994.

[25] G. Bjelakovic, D. Nikolova, and C. Gluud, "Antioxidant supplements to prevent mortality," JAMA, vol. 310 , no. 11 , pp. 1178-1179, 2013.

[26] E. Jansen, D. Viezeliene, P. Beekhof, E. Gremmer, and L. Ivanov, "Tissue-specific effects of vitamin e supplementation," International Journal of Molecular Sciences, vol. 17, no. 7, 2016.

[27] F. E. Martin-Cano, C. Camello-Almaraz, D. Acuña-Castroviejo, M. J. Pozo, and P. J. Camello, "Age-related changes in mitochondrial function of mouse colonic smooth muscle: beneficial effects of melatonin," Journal of Pineal Research, vol. 56, no. 2, pp. 163-174, 2014. 